\title{
Primitivism About Intrinsicality
}

\author{
ALEXANDER SKILES \\ Université de Neuchâtel
}

This is a shortened version of a chapter to appear in Companion to Intrinsic Properties (ed. R. Francescotti, De Gruyter), which will contain classic papers by Rae Langton and David Lewis, Peter Vallantyne, Brian Weatherson, and Stephen Yablo, as well as original contributions by David Denby, Maya Eddon, Michael Esfeld, Carrie Figdor, Vera Hoffmann-Kolss, Dan Marshall, Robert van Gulick, and Gene Witmer. **Please only cite the final, published version**

\section{Introduction}

A reductive analysis of intrinsicality-one that comprehensively, compactly, and non-circularly characterizes what intrinsicality is in metaphysically more fundamental terms - has appeared tantalizingly close at hand to many philosophers. This optimism is perhaps most clearly displayed by the increasingly complicated attempts at reductive analysis offered up in the philosophical literature, and the increasingly sophisticated conceptual resources introduced to state them. (For evidence, just flip through the pages of the book you are now holding.) One hope behind the optimism is that with a reductive analysis one can clarify intuitive judgments about intrinsicality, which David Lewis says are "absurd" to totally dispense with (1983, p. 197), and Theodore Sider deems "as fit a foundation for philosophical theorizing as we can reasonably demand" (1993, p. 2). Another hope is to make more precise the countless distinctions and disputes in philosophy that crucially rely up some antecedent grasp of the notion. And so the already substantial literature cataloguing and evaluating reductive analyses of intrinsicality churns on, showing no signs of slowing (let alone stopping).

But however nice it would be to posses one, the widespread faith that a reductive analysis of intrinsicality is forthcoming - or even that one existsis becoming increasingly difficult to square with the situation on the ground. Attempt after attempt to produce one has met with a battery of recalcitrant cases. Worse, "no-go" results have emerged demonstrating that a number of popular approaches are bound to fail as well. (For evidence, just flip through the pages of the book you are not holding.) And yet there has been scant discussion of why one should be thought to be attainable, or even why taking 
intrinsicality to instead be reductively unanalyzable should be avoided. When a philosophically central notion has revealed itself to be resistant to reductive analysis, making peace with taking it as primitive is often considered to be a live option. Why believe intrinsicality to be any different? Arguments against taking this as a live option have not been articulated or defended anywhere in the literature. Indeed, it is rarely mentioned even as an option (let alone a live one); nor has it been discussed what a viable account of intrinsicality along these lines could, or should, look like.

The goal of this chapter is to explore the prospects of the view I shall call primitivism about intrinsicality, and offer a limited defense. A key aspect of this defense consists simply in clarifying what it would be, exactly, to claim that intrinsicality is "primitive". As we will see, objections to primitivism that seem decisive at first glance turn out to be far less convincing once this notion has been clarified. Doing so is the task of sections 1-3. In section 4, I then consider several arguments for primitivism. And finally, in section 5, I consider several arguments against it.

\section{What is primitivism about intrinsicality?}

As I understand it, primitivism about intrinsicality is the conjunction of the following two theses about intrinsicality:

(PI-1) There are at least some positive mind-independent truths about which properties are intrinsic to which things. ${ }^{1}$

(PI-2) At least some notions of intrinsicality about which there are positive mind-independent truths are reductively unanalyzable (i.e., primitive).

Reductionists about intrinsicality, in contrast, accept (PI-1) yet reject (PI-2). One might weaken (PI-2) slightly, in order to classify as primitivists those who refuse the need to supply a reductive analysis when using intrinsicality for some theoretical purpose. One might also classify as primitivists those who are either doubtful or agnostic about the prospects of supplying one, yet who do not foreclose the possibility. Nonetheless, it is most interesting to see how primitivism and reductionism fare when considered at full strength; thus I will commit the primitivism to (PI-2), and the reductionist to its denial, as it is stated here.

With the restriction in (PI-1) to positive mind-independent truths, I wish to contrast primitivism with two rival views.

\footnotetext{
Here and elsewhere I will suppress extension of what I say to relations, although what I say about properties can be applied to relations straightforwardly.
}

2 ALEXANDER SKILES 
One is eliminativism about intrinsicality: the thesis that there are no truths about intrinsicality beyond 'negative' truths (such as that it is not intrinsic to the Eiffel Tower that it is made of iron, not intrinsic to my body that it has more than one part, and so forth). The eliminativist might maintain this as a (rather surprising) contingent matter of fact. More likely, she maintains this because she believes there to be something defective with the very concept of intrinsicality itself, or with the platitudes used to convey what the concept is. ${ }^{2}$ The eliminativist may agree, though, with the primitivist regarding (PI-2): to believe that there are no positive facts about intrinsicality is compatible with believing that intrinsicality is reductively unanalyzable. Nonetheless the two part ways with respect to (PI-1).

The other view primitivism is to be contrasted with is what one could call non-objectivism about intrinsicality. One version, subjectivism about intrinsicality, holds that whether a given property is intrinsic to a thing is grounded in facts about the subjective responses that tend to be elicited whenever one is confronted with this thing's exemplification of that property (whether it be empirically, or on the basis of a priori reflection). Another version, which I call pragmatism about intrinsicality, instead holds that facts about intrinsicality are grounded in facts about what our practical or epistemic interests in making intrinsicality classifications happen to be at the moment. ${ }^{3}$ Thus far, the non-objectivist may agree with the primitivist about (PI-2): to believe that facts about intrinsicality are grounded in facts about our subjective responses or our interests is compatible with denying that facts of the first kind just are, let alone reduce to, facts of the second kind. ${ }^{4}$ Nonetheless, presumably any reasonable account of what it is for a truth to be mind-independent would entail that the primitivist and the non-objectivist part ways with respect to (PI-2).

The primitivist, then, states that there are some positive mind-independent truths that feature some reductively unanalyzable notion of intrinsicality. But before turning to what might be meant by "reductively unanalyzable", what do I mean by a "notion"? The idea is best conveyed with an example. Take the predicate "is true" (as it is used in "The sentence 'Obama is a Democrat' is true") and the sentential operator "it is true that" (as it is used in "It is true that Obama is a Democrat"). I slice notions finely: although the predicate and

Eliminativism is discussed even less frequently than primitivism is, but the view is given voice on occasion. For instance, Daniel Dennett seems to express the view when he writes that "[i]f even such a brilliant theory-monger as David Lewis can try and fail, by his own admission, to define the extrinsic/intrinsic distinction coherently, we can begin to wonder if the concept deserves our further attention at all" (1988, p. 67).

3 Sometimes the introduction of pragmatic elements is indirect. Taylor (1993), for instance, defends pragmatism of roughly this sort about Lewis's (1983b) distinction between natural and abundant properties, which Taylor then uses to analyze facts about intrinsicality.

4 See section 2, 4.2, and 5.3 for more discussion of grounding, reduction, and the relation between them. 
the sentential operator both allow one to express statements about truth, the two nonetheless express different notions of truth in my sense. Moreover, if (suppose) one thought that truth were reductively unanalyzable, this does not yet settle which these notions (if either) is to be taken as primitive. One view takes only "is true" to express a primitive alethic notion, and takes the notion expressed by "it is true that" to be reductively analyzable in terms of it. A second view says the opposite. A third takes them both as primitive. And the fourth takes the two to be reductively analyzable with other alethic notion(s) altogether. Primitivists about intrinsicality face similar theoretical choices about which notions of intrinsicality to take as primitive, and which way they choose has subtle, and often important, implications for the tenability of the resulting view. I shall explore these choices in more depth in section 3 .

\section{What is it for a notion of intrinsicality to be primitive?}

In a now classic article that helped commence the contemporary literature on intrinsicality, Lewis (1983) presented two counterexamples to an attempt at reductive analysis proposed by Roderick Chisholm (1976) and further refined by Jaegwon Kim (1982). The Chisholm-Kim account characterizes what intrinsicality is in terms of notions that are purely modal and mereological. A reductive analysis of what it is for a property to be intrinsic-or what Lloyd Humberstone (1996) has dubbed global intrinsicality - which maintains the spirit of the Chisholm-Kim account is the following:

(CKG) $\quad F$ is an intrinsic property iff: $F$ is a property, and necessarily, if some $x$ has $F$, then it is possible that (i) $x$ has $F$ and (ii) every contingent existent is a part of $x$.

(If $x$ satisfies condition (ii) above, then it is said to be lonely.) In contrast, a reductive analysis of what it is for a property to be intrinsic to a particular thing-or what Humberstone calls local intrinsicality - which maintains the spirit of the Chisholm-Kim account goes like this:

(CKL) $\quad F$ is an intrinsic property of $x$ iff: $x$ has $F$, and it is possible that both (i) $x$ is $F$ and (ii) $x$ is lonely. ${ }^{5}$

In rough slogan form: intrinsicality is nothing beyond whether a property can be had by lonely things.

5 For the time being, I will suppress the question of whether either the having of a property, or the having of a property intrinsically, must also be relativized (at least) to a time and/or a possible world, although the issue will reemerge in an important way in section 4.2.

4 ALEXANDER SKILES 
My concern in the following subsection is neither with Lewis's particular counterexamples to the Chisholm-Kim account, nor is it to chart the many refinements that have been introduced in order to evade them. Rather, I want to ask: what are some of the necessary conditions for statements like (CKG) and (CKL), when offered as reductive analyses, to be correct? My answer falls far short of a reductive analysis of reductive analysis. And moreover, some reductionist may well disagree with what I take some of the necessary conditions to be (or even whether they are saddled with what $I$ have called a "reductive analysis"). Nonetheless, my answer will comport well with what the primitivists and the reductionist seem to take to be at stake in the dispute between them. ${ }^{6}$

A notion of intrinsicality is reductively analyzable just in case there exists a reductive analysis, which one might think of as a type of proposition that $(\mathrm{CKG})$ and (CKL) both purport to state. (On occasion I will use "reductive analysis" to refer to the activity of attempting to produce reductive analyses, although this is shorthand.) If there exist no other notions of intrinsicality beyond the notions of global and local intrinsicality expressed above, then there exists a reductive analysis of every notion of intrinsicality if $(\mathrm{CKG})$ and $(\mathrm{CKL})$ succeed at stating reductive analyses. ${ }^{7}$ So the key question is: what is necessary for $(\mathrm{CKG})$ and $(\mathrm{CKL})$ to state reductive analyses?

It is by now well known that it is necessary, but not sufficient, that each substitution instance of " $F$ " and " $x$ " in (CKG) and (CKL) should yield a pair of necessarily true sentences. For if this were also sufficient, then reductively analyzing intrinsicality would be far too easy: " $F$ is an intrinsic property" is necessarily equivalent in truth-value to "an omniscient being would know that $F$ is an intrinsic property", " $F$ has this property" (where "this property" directly picks out the property of being an intrinsic property, perhaps via a mental act of ostension), and " $F$ is an intrinsic property". But clearly none of these express reductive analyses. The difficult task for a full-blown account of what reductive analysis would be to specify what more is involved beyond necessary covariation in truth-value. Although I have no sufficient condition to provide, I assume that the following two conditions are at least necessary.

Although there is some literature on the nature of reductive analysis (and more generally of the nature of what I call metaphysical analysis. to contrast it with 'conceptual analysis'see below), the issue has received relatively little direct attention given how often it crops up in philosophical discussions. A concept similar in at least some respects to what I am calling "reductive analysis" is explored by Fine (1994), King (1998), Dorr (2004, 2005, 2008, manuscript), Melia (2005), Rayo (2013), Schroeder (2005), and Wedgwood (2007), but there are stark differences separating how these authors articulate the concept and also separating them from how I articulate the concept here. Space prevents a full comparison, although I hope to provide one in future work.

Although it is not uncommon to say that a sentence expresses a reductive analysis even if it is unsuccessful. Those who choose to speak this way should insert "true" in from of every occurrence of "reductive analysis" in this chapter. 
First, for (CKG) and (CKL) to state reductive analyses, there must be an identification, so to speak, between the 'aspect of reality' the analysandum sentence concerns and the 'aspect of reality' the analysans sentence concerns. This requirement-call it the identification requirement-is easiest to grasp when the notion targeted for reductive analysis is a property. For (CKG) to state a reductive analysis, e.g., it must successfully express which property the higher-order property being an intrinsic property really is. As King puts it, (CKG) cannot merely "[say] that the property in question is related to this or that property in certain ways" $(1998$, p. 177 fn. 26). And since being an intrinsic property is intuitively not identical to the property would be known to be an intrinsic property by an omniscient being, we can then say that the sentence " $F$ is an intrinsic property iff: an omniscient being would know $F$ to be an intrinsic property" fails to state a reductive analysis, as desired. ${ }^{8}$

Although the identification requirement is clear enough when a property is the targeted notion, it is difficult to state what it involves when some other kind of notion is involved - that is, without straying into very controversial metaphysical matters that I ultimately wish to stay neutral towards. Many would want to maintain that one can reductively analyze the notion expressed by "it is true that", to recall an earlier example, without committing oneself to there being a strange (operator-shaped?) entity that serves as a relatum for the identification. But how could one apply the identification requirement to this example otherwise?

For sake of concreteness, and since the discussion to follow will largely not hinge on such matters, I shall work with my own views about them and construe the identification requirement as follows. Suppose that if a sentence, " $p$ ", is true, then it picks out an obtaining fact, which I symbolize as: [ $p]$. Although I assume that there are facts, I will not assume that the predicates, sentential connectives, or other expressions occurring in " $p$ " also pick out constituents of $[p]$, or even that $[p]$ has any other kind of internal structure. However, I will assume that a necessary condition for a statement like (CKG) or (CKL) to express a reductive analysis is that for any uniform substitution of " $x$ " and " $F$ " with names for particular objects and particular properties, the resulting analysans sentence, if it is true, picks out one and the same fact as the resulting analysandum sentence. ${ }^{9}$

8 I am taking for granted that properties are not individuated merely by sets of their actual and possible instances, and to at least to this extent are not too "coarse-grained".

9 When are 'two' facts identical? My own view is that there are no interesting principles of fact individuation that apply across the board, even though there may well be interesting, non-trivial cases of fact identity. If there were such principles, then presumably they would need to be 'fine-grained' enough so that $[a$ is intrinsically $F]$ and [an omniscient being would know $a$ to be intrinsically $F$ ] would count as distinct, yet coarse-grained enough that seemingly uncontroversial instances of reductive analysis like [George Clooney is a bachelor] and [George Clooney is an adult unmarried male] are identical. For alternative

6 ALEXANDER SKILES 
Alongside the identification requirement, I also assume that the embedded analysans sentence in a successful reductive analysis must also express only notions that are in some sense metaphysically more fundamental than the notion targeted for reductive analysis. Spelling this requirement out in full detail also leads us into vexed matters I wish avoid. Nonetheless, I take what I call the relative fundamentality requirement to involve a couple constraints. Notably, both of these constraints are compatible with a reductive analysis also satisfying the identification requirement.

\section{The grounding constraint:}

The first upshot makes use of the concept of metaphysical grounding: the relationship by which one collection of facts is said to non-causally 'derive' from, hold 'wholly in virtue of', or 'result' from another collection of facts. Since the concept has performed such a prominent role in recent attempts at reductively analyzing intrinsicality, I will presuppose a basic familiarity with it here. $^{10}$

To help you get a feel for the grounding constraint, suppose that (CKL) is true. And consider a sentence with the same logical structure as an instance of (CKL): that is, any sentence with the form " $p$ iff: $(q \wedge r)$ ". And say that a sentence with this form expresses an instance of this reductive analysis if and only if it is both true and is the result of uniformly substituting " $x$ " and " $F$ " in (CKL) with names for particular objects and properties. Then (CKL) meets the grounding constraint only if for every instance of (CKL), the following two conditions are met: $(i)$ the fact picked out by the analysandum sentence, " $p$ ", is grounded in the collection of facts picked out by " $q$ " and " $r$ " and (ii) neither fact in this collection is itself even partially grounded in facts picked out by sentences that express the relevant notion of local intrinsicality. Thus if (CKL) states a reductive analysis, then the fact that being made of iron is intrinsic to the Eiffel Tower is grounded in the collection consisting of the following two facts, neither of which are themselves even partially grounded in facts involving the relevant notion of local intrinsicality: namely, [The Eiffel Tower is made of iron] and [It is possible for the Eiffel Tower to be both be made of iron and be lonely].

I have only stated the constraint for putative reductive analyses that take the conjunctive form that (CKL) does. Nonetheless, it can be generalized in obvious ways to putative reductive analyses that differ from (CKL) in logical structure. ${ }^{11}$

conceptions of reductive analysis that allow for identification without fact individuation, see Dorr (manuscript) and Rayo (2013).

10 For discussion, see Witmer et al. (2005), Rosen (2010), and Bader (forthcoming).

11 One natural idea would be to 'read off' these principles from a sentence's syntactically circumscribed truth-conditions. So, for instance, one might say that if " $p$ iff: $(q \vee r)$ " is an 


\section{The essence constraint:}

The second way that an analysans sentence must express 'metaphysically more fundamental' notions involves the concept of a collection of truths that describe a notion's essence. These are the true sentences that hold in virtue of that notion's nature or identity, and as such exhaustively characterize what that notion is (as opposed to how it is). ${ }^{12}$

Some terminology: supposing for sake of simplicity that it is unique, let $\Sigma$ be the collection of truths about some notion $v_{1}$, and say that a notion $v_{2}$ is a constituent of the essence of $v_{1}$ just in case it is expressed by a constituent of a truth in $\Sigma$. (So for example, if $\Sigma$ collects every truth about the essence of the set $\{$ Socrates\}, including "Socrates is a member of \{Socrates\}", then the terms "Socrates", "is a member of", and " $\{$ Socrates\}" express constituents of the essence of \{Socrates\}.) The essence constraint, then, is the claim that if $v_{1}$ is reductively analyzable in terms of $v_{2}$, then $v_{1}$ is not itself a constituent of the essence of $v_{2}$. In other words, a notion cannot figure into the essence of notions it is reductively analyzable in terms of.

The essence constraint has at least two prima facie motivations. First, it is plausible to think that if $\boldsymbol{v}_{1}$ is reductively analyzable in terms of $\boldsymbol{v}_{2}$, then $\boldsymbol{v}_{2}$ is automatically included in truths about what $\boldsymbol{v}_{1}$ is. Intuitively, a collection of truths that fails to include "every bachelor is an unmarried male" would fail to include an important truth about the essence of bachelorhood. And yet it is also plausible that constituents of a notion's essence help to determine nontrivial individuation conditions for that notion, and that a notion cannot help determine non-trivial individuation conditions for that which determines its own. ${ }^{13}$ From the previous claims, the essence constraint follows.

A second (and more mundane) motivation behind the essence constraint is simply that philosophers commonly reason about reductive analysis as if it were true. For instance, philosophers who claim to analyze what necessity and possibility are in terms of facts about the 'causal powers' of actual things (e.g., the causal power of a cube of sodium chloride to dissolve in water) will often immediately add that the proposed account is not offered as a reductive

instance of some reductive analysis, then $[p]$ is either grounded in $[q]$ or grounded in $[r]$, that if " $p$ iff: $\exists x \Phi(x)$ " is an instance of reductive analysis, then for any " $a$ " with a referent for which " $\Phi(x)$ " is true, $[p]$ is grounded in $[\Phi(a)]$, and so on. Complications emerge when considering analysans sentences with main connectives like "it is possible that" or "it will always be the case that", since it is a vexed issue whether such sentences are grounded at all, and how they are grounded if so. Unfortunately I cannot pursue the matter here.

12 For influential contemporary discussions, see Fine $(1994,1995)$ and Lowe (1998). In these works, both Fine and Lowe focus on the concept of objectual essence, which figures into answers to questions like "What is it to be Socrates?". However, Correia (2006) has shown that there is in addition the concept of generic essence, which figures into answers to questions like "What is it for a proposition to be true?". I primarily have generic essence in mind, extended to sentences that need not have subject-predicate logical structure.

13 See Lowe (2012), who provides an argument for these two claims.

8 ALEXANDER SKILES 
analysis. Why? Because they also take the collection of truths about a causal power's essence to include truths about which effects they would or could produce across actual and non-actual circumstances. ${ }^{14}$ Since these are notions of necessity and possibility, the implicit assumption these philosophers are making is that notions of causal power cannot reductively analyze them since they figure into the essence of causal power. This, of course, is just a specific instance of the essence constraint.

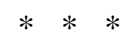

One might choose to add further constraints - requiring that the analysans sentence not just be a mere disjunction of all possible scenarios in which the analysandum sentence is satisfied, say. But only the previous two constraints will play a role in what follows.

Note, though, that I have not assumed that a reductive analysis is required to satisfy cognitive constraints of any kind. For instance, I do not assume that a reductive analysis must be informative, or that epistemic access to it either can or must be a priori, or that a reductive analysis state a conceptual truth (in any of the myriad senses of "conceptual truth" found in the literature). As I understand it, the reductionist about intrinsicality is only committed to the metaphysical analyzability of intrinsicality - not with how it is conceptually represented in either thought or talk, nor with how one could gain evidence for or against this or that reductive analysis.

\section{Which notions of intrinsicality are primitive?}

Even though primitivists all agree that at least some notion of intrinsicality is primitive, nonetheless there remains the question of which notions these are. Reductionists face a similar dispute: which notions of intrinsicality are not reductively analyzable in terms of the other notions of intrinsicality, and thus serve as the "entry points" for the reductive account? Yet the question that faces the primitivist seems more pressing. For an unwise choice of primitives may expressively impoverish the primitivist's account if they are too few or of the wrong kind; it may render it ideologically unparsimonious if there are instead too many and redundancies emerge.

Rather than take on the general question of which notions the primitivist should take as primitive, in this section I merely sketch out some options that deserve consideration. How they differ will often an impact on the prospects of primitivist accounts.

14 Cf. Jacobs (2010), esp. 239. 
Which intrinsicality platitude(s) is the notion supposed to satisfy?

As Weatherson and Marshall (2012) note, one might distinguish between a number of non-equivalent varieties of intrinsicality, each of which may be naturally associated with a given platitude about intrinsicality. Thus one may distinguish properties had independently of outside forces (cf. Ellis 1991) or independently of external environment more generally (cf. Figdor 2008). Or properties that any pair of possible duplicates must share (cf. Lewis 1983b). Or properties had wholly in virtue of the way that a thing is in and of itself (cf. Vallantyne 1997). Or even still, properties the ascription of which only concern a thing and its proper parts, rather than its relations to other things (cf. Francescotti 1999).

The primitivist has a choice. On one hand, she may take some or all of these as imperfect glosses on a single common primitive notion. Or instead, she may take some or all of these to express distinct notions of intrinsicality. And if one goes the latter route, she must make the further choice about which one or more of these notions to take as primitive. Call a pluralist any primitivist who takes two or more of these platitudes about intrinsicality to each be associated with at least one notion of intrinsicality, and call a monist any primitivist who is not also a pluralist.

\section{Local or global intrinsicality?}

As was noted before, it is now common to distinguish the claim that the property being less than a kilometer from the Eiffel Tower is intrinsic to the Eiffel Tower (a seemingly true claim about local intrinsicality) from the claim that it is intrinsic, full stop (a seemingly false claim about global intrinsicality - a tourist climbing the Eiffel Tower may have this property, yet not intrinsically).

Now, it has become standard to take global intrinsicality to be reductively analyzable in terms of local intrinsicality. ${ }^{15}$ Yet even so, there is no logical contradiction in taking some primitive notions to express notions of global intrinsicality, while others express primitive notions of local intrinsicality instead. For instance, suppose the primitivist believes that for a property to be globally intrinsic is just for it to necessarily be locally intrinsic whenever exemplified. Nonetheless, she may still deny that each and every fact about global intrinsicality is grounded in facts about local intrinsicality (therefore violating the grounding constraint from section 3). Or she may maintain that some truths about the essence of primitive notions of local intrinsicality include at least some notions of global intrinsicality (therefore violating the essence constraint from section 3). This is an issue about which primitivists may genuinely debate.

15 For instance, see Witmer et al. (2005) and Bader (forthcoming). 


\section{Which grammatical categories express the relevant notions?}

Suppose that there is some primitive notion of local intrinsicality. Even so, there might still be a number of ways to express the grammatical structure of sentences expressing this notion, and thus many possible primitive notions of local intrinsicality to choose from. One may take it to be expressed with a relational predicate (“...is intrinsic to..."). Or one may take it to be expressed with a sentential operator that takes a sequence of things and a formula and yields a closed sentence ("it is intrinsic to...that..."). Or one may take it to be expressed with an adverbial modifier that combines with one predicate to yield another (“...intrinsically"). Or one may take it to be expressed with a monadic predicate of facts ("...is an intrinsic fact").

Sorting out which of these options to go in for is an important theoretical task for the primitivist, for at least two reasons. First, some of these primitive notions seem to bring ontological commitments along with them that the primitivist might want to reject (e.g., to properties, or to facts). Second, it is not at all obvious whether every statement about local intrinsicality phrased in terms of one of these notions can be expressed with a statement phrased in terms of any other, and thus whether some of these notions have more or less expressive power relative to the others.

\section{Are the notions comparative or non-comparative?}

A final decision point for the primitivist is whether to take the primitive notions of intrinsicality to be non-comparative - stating that $F$ is intrinsic, or that $F$ is intrinsic to $x$, simpliciter-rather than comparative. Now although comparative notions of intrinsicality are underexplored, they seem coherent, and there is no obvious reason why the primitivist could not make use of them. ${ }^{16}$ One might take as primitive the notion of $x$ 's being $F$ intrinsically to a certain degree. Or one might take as primitive the notion of $x$ 's being $F$ intrinsically more, or less, or as much as, a given $y$ 's being $G$ intrinsically (where $x$ may be identical to $y$, or $F$ may be identical to $G$ ). Nothing prevents the primitivist, qua primitivist, from taking comparative notions as primitive (or not).

$$
* * *
$$

The primitivist thus has considerable theoretical work to do. (I have not even considered related questions about whether any notions of extrinsicality are to be taken as primitive, and which to take as primitive if so.)

16 Figdor (2008, p. 699) briefly notes that her reductive analysis and others that have been recently proposed allow for local intrinsicality to be had in degrees, but does not explore the possibility further. 


\section{Arguments for primitivism about intrinsicality}

So far I have focused on how to understand primitivism. The remainder of this chapter considers what reasons might be brought to bear for or against it. In this section, I discuss two families of arguments for primitivism (although without foreclosing the possibility that other arguments might be devised).

\subsection{Arguments that appeal to past track records of failure}

It is completely uncontroversial to observe that despite repeated attempts, no attempt at reductively analyzing intrinsicality garners even majority support among the practitioners. It is only slightly more contentious to claim that for any extant account there are (at least prima facie) decisive counterexamples, amongst other obstacles. Rather than rehash the many articles chronicling these obstacles, let us suppose they stand and see what follows. ${ }^{17}$

The fact that these obstacles do stand is of course consistent with the existence of a hitherto unknown reductive analysis. To construct a genuine argument for primitivism on the basis of this fact, the primitivist must either reason inductively from it (by arguing that there is a large and representative enough sample of repeated failures that we can conclude that every possible attempt would result in failure too), or reason abductively from it (by arguing that the non-existence of a reductive analysis is the best explanation for why we have yet to discover one).

And even then, such reasoning might come in one of two broad varieties. The appeal can be either restricted, by focusing on the past track record of failure to reductively analyze intrinsicality in particular; or unrestricted, by focusing on the past track record of failure to reductively analyze any notion of philosophical interest.

For instance, a restricted inductive track record argument for primitivism incorporates as a premise the claim that the sample of past attempts is both large enough (since there have been many such attempts) and representative enough (since reductive analysis has been pursued under a diverse array of approaches and have employed a diverse array of notions). This is in contrast to Timothy Williamson, who offers an unrestricted abductive track record argument when he asserts that "[t]he pursuit of analyses is a degenerating research programme" that had its origin "in great philosophical visions" such as Russell's logical atomism and the principle of acquaintance, but now "the

17 Weatherson and Marshall (2012), Marshall (2012), and Marshall (forthcoming- $a$ ) are particularly thorough surveys of problems with the various attempts. Until recently, my own view was that intrinsicality could be reductively analyzed in terms of notions of grounding and mereology, roughly along the lines of the proposals found in Rosen (2010) and Bader (forthcoming). However, recent criticism of such accounts due to Marshall (forthcoming- $b$ ) now give me pause.

12 ALEXANDER SKILES 
philosophical visions that gave it a point are no longer serious options" that could sustain its pursuit (2000, pp. 31-32).

How should the reductivist respond, to start with, to restricted arguments for primitivism (assuming, again, the reductivist grants that no successful account has been developed)? There are a number of strategies; each of these could be construed either as a reason to doubt that the past track record of failures is large and representative enough of a sample to support a strong inductive generalization, $o r$ as an alternative explanation to the primitivist's non-existence hypothesis.

First, the reductivist might point out that although notions of intrinsicality have been used in philosophical conversations for centuries, the academic industry devoted to reductively analyzing intrinsicality is comparatively new, beginning in earnest only during the 1980s. Perhaps that industry has only scratched the surface of the number and variety of possibilities that have some viability.

A second kind of response is this. There is no agreement about what a reductive analysis of intrinsicality should look like. However, there is a growing (although not unanimous) consensus that one must inevitably go beyond the "quasi" or "broadly logical" notions of logic, modal, mereology, set theory, and property exemplification in order to state a proposal that has any hope of success. ${ }^{18}$ The predominant diagnosis is that one must in addition appeal to notions that hyperintensionally distinguish intrinsic properties from those they may be necessarily co-exemplified with. The disagreement among reductionists is what the source of this hyperintensionality. Some claim the source has to do with naturalness (Lewis 1983b; Langton and Lewis 1998) others grounding and fundamentality (Witmer et al. 2005; Trogdon 20009; Rosen 2010; Bader forthcoming), and still others the notion of an event or state of affairs "consisting in" another (Francescotti 1999). Reductionist may well note that the project of polishing the resources needed is comparatively new as well, and argue that for all the primitivist has shown, further polishing might be all that is needed for success.

A third kind of response goes as follows. As I said in section 3, there is also a growing consensus that there are a variety of slightly divergent notions of intrinsicality, and slightly divergent platitudes about what intrinsicality amounts to, that have been targeted by philosophers for reductive analysis. The reductivist may grasp hold of this observation, and note that the presence of a past track record of failure may have more to do with mismatch between the reductive analysis offered on one hand and the platitude and/or notion

Talk of "quasi" or "broadly logical" notions in this context, and argument that any such account must fail, comes from Sider (1996) and Marshall (2009), respectively. For dissent, see Vallentyne (1997), Yablo (1999), Denby (2006); and Hoffmann (2010); for discussion of whether the accounts contained in these works are both broadly logical and successful, see Weatherson and Marshall (2012) and Marshall (forthcoming- $a$ ). 
targeted. Or she might argue that it has more to do with the assumption that there is a single notion of intrinsicality that all other notions of intrinsicality are reductively analyzable in terms of. It may just be too early to tell whether reductively analyzing different notions of intrinsicality independently might meet with more success (or so the reductionist might claim).

Although none of these obstacles to restricted track record arguments for primitivism are decisive, they are serious. Thus the primitivist who wishes to advance such arguments must grapple with them.

In contrast, I will have much less to say about unrestricted arguments for primitivism, since the literature dealing with these arguments is far more extensive than can be surveyed here..$^{19}$ But here are a couple notes of caution for the would-be proponent. First, those who wish to motivate primitivism, at least in part, by appeal to its reductive power (cf. section 4.2) are undermined as much by unrestricted track record arguments as the reductionist. Second, those who have advanced unrestricted track record arguments often argue on the basis of cognitive constraints on reductive analysis. Williamson, for example, points to failures of "concept identity" (2000, p. 30). Others argue instead that the past track record of reductive failure is best explained by unreliability of a priori intuition as a reliable source of evidence. However, the reductionist need not require such cognitive constraints be satisfied for reductive analysis (given the conception of reductive analysis I sketched in section 2 , at least).

\subsection{Arguments from the inaptness of notions required for reductive analysis}

Reductionists have employed a large collection of exotic notions in attempts to reductively analyze intrinsicality, notions that have themselves been taken as primitive (see p. 13 for a short, slightly less than comprehensive list). This observation suggests another way to support primitivism. One could argue that if intrinsicality were reductively analyzable, it would have to be done so at least partly in terms of such-and-such specific notions, and then argue that these notions are inapt to play the required reductive role.

Recalling the discussion from section 2, the primitivist could show that a given notion is inapt in at least one of two ways. First, the primitivist could show that one of these notions is better reductively analyzed in terms of intrinsicality rather than vice versa. Given the grounding constraint, it would follow that the relevant notion of intrinsicality is not reductively analyzable in terms of this inapt notion. Second, the primitivist could show that the notion is inapt by showing the relevant notion of intrinsicality is a constituent of the inapt notion's essential nature. Neither of these kinds of arguments would demonstrate that one could not illuminate what intrinsicality in terms

19 For a small sample, see many of the essays in DePaul and Ramsey (1998). 
of these inapt notions, nor that one cannot provide informative necessary and sufficient conditions of the former in terms of the latter. But either argument would demonstrate that such an account must fall short of reductive analysis. Given the supposition that any viable successor account must employ these inapt notions, primitivism about intrinsicality would follow.

Are there any such notions that could suit the primitivist's purposes? The most obvious candidate is parthood and related notions from mereology. For nearly every major purported reductive analysis of intrinsicality appeals to mereological notions. These include those that appeal to the technical notions of loneliness and independence of accompaniment (e.g., Langton and Lewis 1998, Witmer et al. 2005, Trogdon 2009). Or the notion of an isomorphism that preserves perfectly natural properties and relations among a thing's parts (e.g., Lewis 1986). Similarly when one looks at the details of accounts that set mereological restrictions on the ways of modally recombining properties and property instantiations, which are taken to generate the set of intrinsic properties (e.g. Weatherson 2001 and Denby 2006). Or that set mereological restrictions on the kinds of objects that cannot be 'removed' from or 'ignored' in a thing's environment without also varying that thing's intrinsic properties (e.g., Vallentyne 1997, Yablo 1999, and Figdor 2008). Or that set mereological restrictions on the kinds of facts that reduce and/or ground facts about that thing's intrinsic properties (e.g., Francescotti 1999, Skow 2007, Rosen 2010, and Bader forthcoming).

Now, I have no ironclad argument that one cannot reductively analyze intrinsicality without some mereological notion. But it is difficult (at best) to think of how a viable reductive analysis would go.

So, then, may the primitivist convincingly argue that for each notion of mereology, the collection of truths that characterize its essence must express some notion of intrinsicality? As I will not argue, indications that the correct answer is yes seem quite strong.

It is routine for philosophers to make appeal to truths about intrinsicality when attempting to convey truths about the essential nature of central mereological notions. Fine observes when introducing "the intuitive notion of part" that "[w] hen one object is a part of another, there is a sense in which it is in the other-not in the sense of being enclosed by the other, as when a marble is in an urn, but more in the sense of being integral to the other" (2012, p. 560, his emphasis). Although Fine does not characterize what 'integrality' consists in, it is tempting to take it as expressing a notion of intrinsicality. Similarly, Theodore Sider observes that parthood seems constrained by a principle that he dubs "the inheritance of intrinsicality": if a property, $F$, is intrinsic, then the property having a part that has $F$ is also intrinsic. In this sense at least, part of what it is to be a part is for its intrinsic nature to be 
"reflected in the whole" $(2007$, p. 70$){ }^{20}$ For a third example, one that returns us to the intrinsicality literature, Stephen Yablo notes the following three connections between parthood and intrinsicality, and claims them to be "nonaccidental" and "as de jure as anything":

- If $x$ is part of $y$, then $x$ cannot change intrinsically without $y$ also changing intrinsically.

- If $x$ is part of $y$, then $x$ and $y$ have a region of intrinsic match.

- If $x$ is properly part of $y$, then $x$ and $y$ have intrinsic differences (Yablo 1999, p. 482).

(One might add to Yablo's list. For example, it seems "nonaccidental" and "as de jure as anything" as well that if $x$ is a part of $y$, then this is an intrinsic fact about the plurality consisting of $x$ and $y$. But let us settle for Yablo's list for now.) By "nonaccidental", Yablo explicitly tells us that he does not mean contingent. Contrasting what he takes to be "de facto" connections between intrinsicality and the Lewisian notion of naturalness, Yablo claims that there is "nothing in the nature of intrinsicness" that prevents this connection from coming apart (ibid. p. 481), and adds that this would strike us as so even if the connection happens to hold with necessity (ibid, fn. 4).

Although there appears to be a strong intuitive case for the claim that notions of mereology violate the essence constraint, the reductionist might resist. First, the reductionist could of course just reject the essence constraint, although doing so would require explaining away the prima facie support for it. Second, the reductionist might claim that although the connections state necessary truths about the relevant notions of mereology, they do not state essential truths about them. Third, the reductionist might claim that although these connections do state essential truths, they state essential truths about intrinsicality rather than about notions of mereology.

May the primitivist instead argue that the notions of mereology relevant for reductively analyzing are inapt because they are reductively analyzable in terms of notions of intrinsicality, and not than vice versa? Here again, the primitivist seems to be able to make a surprisingly strong case. I will briefly consider five attempts to reduce parthood in terms of intrinsicality. Although I cannot explore all the issues they raise here, or whether any variation of one of these accounts is successful, they are worth the primitivist's effort to

Similarly, Fine claims that "[i]n the case of the 'intrinsic' character of a thing - such as its mass and its color-the character of the whole will be some sort of function of the character of the parts" that varies from case to case, and then adds that "I am inclined to regard these various principles as definitive of the form of composition in question. It will lie in the nature of any form of composition to conform to various principles of this sort" (2012, p. 571). 
explore in more detail (although ultimately the last pair of accounts at least initially seem more worth effort than the first three).

First, recall Fine's observation that a part of a thing is in some sense 'in' or 'integral' to the thing it is a part of. Normally when one speaks of a thing, $x$, being intrinsic to another thing, $y$, one takes " $x$ " to range over properties (as, for example, (CKL) does from section 2). But one could remove this restriction, and take " $x$ " to range not only over properties, but rather over objects of any arbitrary ontological category. One might then claim that $x$ is a proper part of $y$ iff: $x$ is intrinsic to $y$, and $x$ is not a property.

Although straightforward, the account has at least one major disadvantage that a thing cannot possibly have any properties as parts. Some might see this as an attractive result. Those who instead wish to evade this result could remove the qualification that $x$ not be a property. But then the difficulty is to distinguish cases in which a thing has a property intrinsically from cases in which a thing has a property merely as a part. For the two cases can come apart. Even if the two-membered set \{Socrates, being a philosopher\} has the property of being a philosopher as a part, the set clearly is not itself a philosopher (let alone does the set have this property intrinsically). Cashing out this distinction without mereological notions will be challenging.

One may instead look to the de jure connections Yablo lists for further insight into how to reductively analyze parthood in terms of intrinsicality. For instance, a second and third attempt start with Yablo's claim that if $x$ is part of $y$, then $x$ cannot change intrinsically without $y$ changing intrinsically as well.

There are at least two ways to read Yablo's claim. One reads it as the claim that intrinsic changes to $x$ necessitate intrinsic changes to $y$. The other instead reads it as the weaker, counterfactual claim that intrinsic changes to $x$ would lead to intrinsic changes to $y$. In a similar fashion, one may attempt to reductively analyze parthood in terms of intrinsicality as follows:

(YP-1) $\quad x$ is a part of $y$ iff: $\square(x$ undergoes intrinsic change $\rightarrow y$ undergoes intrinsic change)

(YP-2) $\quad x$ is a part of $y$ iff: ( $x$ undergoes intrinsic change $\square \rightarrow y$ undergoes intrinsic change)

Although perhaps an account in the vicinity of (YP-1) and (YP-2) could be made to function properly, at minimum it should avoid the following two problems. (And perhaps others: e.g., the implication that a thing which could not undergo intrinsic change trivially counts as a part of every other object!) First, (YP-1) does not specify a necessary condition for parthood, and is thus too strong. Suppose that $x$ is a piece of iron that is actually part of the Eiffel Tower. Clearly, it is possible for $x$ to undergo intrinsic change without the 
Eiffel Tower undergoing intrinsic change. For suppose that $x$ were part of the Hancock Tower instead. Second, (YP-2) clearly does not satisfy a sufficient condition for parthood, and is therefore too weak. Presumably, the closet counterfactual circumstances in which the Eiffel Tower undergoes intrinsic change are circumstances in which at least one of the pieces of iron that is part of it undergoes intrinsic change (say, a slight increase in temperature). Let $x$ be such a part. Then since $x$ would undergo intrinsic change if the Eiffel Tower undergoes intrinsic change, (YP-2) entails that the Eiffel Tower is part of $x$-an absurd result.

The final two accounts I will consider take the more promising route of starting with Yablo's claim if $x$ is part of $y$, then $x$ and $y$ have a region of intrinsic match.

Although Yablo does not further gloss what he means by "having a region of intrinsic match", one might try to flesh it at least in one of the following two ways. The first way brings in Sider's observation that a thing somehow 'inherits' the intrinsic properties of its parts. Although Sider's inheritance principle employs the notion of parthood, a nearby principle does not. Again, let $x$ be a piece of iron that is part of the Eiffel Tower. If being a piece of iron is intrinsic to $x$, it seems not only to be the case that having a piece of iron as a part is intrinsic to the Eiffel Tower. It also seems to be the case that being such that a piece of iron exists is intrinsic to the Eiffel Tower. One does not need to 'look beyond the boundaries' of the Eiffel Tower, or inspect anything other than how the Eiffel Tower is 'in and of itself', to determine that a piece of iron exists.

One may attempt to reductively analyze parthood in terms of intrinsicality by claiming that it is necessary and sufficient if this holds for every property intrinsic to $x$, as follows:

(YP-3) $x$ is part of $y$ iff: for every property $F$, if $F$ is intrinsic to $x$, then the property being such that an $F$ exists is intrinsic to $y$.

To devise an account of proper parthood, one may either add the condition that $x$ and $y$ are distinct, or (to evoke Yablo's third de jure connection, that a thing and its proper parts must have "intrinsic differences") add the condition that there is some property, $G$, that is intrinsic to $x$ even though the property being such that a $G$ exists is not intrinsic to $y$.

Another way to make sense of the phrase "having a region of intrinsic match" that might prove useful in this context reads Yablo's phrase much more literally. It is intuitive to think that things can exemplify properties not simpliciter, but rather only relative to a particular region of space. After all, a checkerboard does not exemplify blackness and whiteness simpliciter, but rather blackness here and whiteness there. 
A tempting thought is that for a thing, $x$, to have a property, $F$, in a spatial region $r$ is just for $x$ to have a proper part, $y$, that is $F$ and exactly occupies $r$. However, a number of philosophers have independently argued that having a part in a spatial region does not imply, and thus does not reduce to, having a proper part that exactly occupies that region. The argue that by applying this distinction, one yields numerous philosophical benefits. ${ }^{21}$

The thought seems directly applicable to reductively analyzing parthood, for if a black piece of the checkerboard is black, square-shaped, felt-covered, and so forth in a square-shaped region, then presumably the checkerboard also has all those properties in that region as well. Generalizing this thought to all the intrinsic properties of a part in some region, one might attempt to reductively analyze parthood as follows:

(YP-4) $\quad x$ is part of $y$ iff: for every property $F$ and every spatial region $r$, if $x$ has $F$ in $r$ intrinsically, then $y$ has $F$ in $r$ intrinsically.

The main challenge for a proponent of (YP-4) will be to motivate the claim that it is possible for an occupant of a region to have properties in that region intrinsically. However, there is an extensive literature defending the claim that a thing can have properties relative to a particular time or possible world intrinsically, and no immediately obvious reason why a proponent of (YP-4) could not co-opt some of these familiar maneuvers. ${ }^{22}$

So far I have only considered whether the primitivist could reasonably attempt to reductively analyze parthood in terms of intrinsicality. But to argue against reductionism, the primitivist must show further that one should reductively analyze parthood in terms of intrinsicality. This would require asking whether one should take parthood to be reductively analyzable at all; if so, one would then need to compare how the primitivist-friendly accounts stack up against reductionist-friendly accounts. What I have tried to show is merely that these two questions are worth pursuing.

\section{Arguments against primitivism about intrinsicality}

The most direct way to argue that a notion is not reductively unanalyzable is (naturally enough) to propose a reductive analysis and then argue that it is successful. But the reductionist might wish to rationally reject primitivism

21 For instance, Parsons (2000; 2004), Sider (2001), and McDaniel (2004) all suggest that the distinction can help explain how extended objects that are either temporally or spatially simple can be also qualitatively heterogeneous. Hudson (2001) uses the distinction to propose a novel solution to the infamous 'problem of the many'.

22 See Eddon (2010) for a recent survey and discussion of the maneuvers available in the case of time-relative property exemplification. 
even if is either agnostic about, or even disbelieves, that any extant account is successful. In this section, I consider two kinds of objection.

\subsection{Primitivism is uninformative}

A common argument wielded against primitivism about any phenomenon is that it fails to do what any viable philosophical account is supposed to do: provide at least some explanatory understanding of the phenomena. ${ }^{23}$ Hence the argument against primitivism about intrinsicality in particular is that by refusing to specifying what intrinsicality is in other terms, the primitivist renders the phenomena of intrinsicality epistemically opaque or inaccessible altogether.

But what is it, exactly, about intrinsicality that primitivism leaves us in the dark about? I do not see how the reductionist can respond that will not also undermine accounts of intrinsicality that are reductive. The reductionist might claim that a primitivist account does not tell us much about which properties are intrinsic, or about which properties are had intrinsically on which occasions. Or the reductionist may claim that primitivism either fails to provide, or altogether precludes, any firm understanding of the essential nature of intrinsicality. But as I have emphasized before (c.f. section 4.1), the vast majority of viable reductionist accounts on offer employ a variety of primitive notions (like naturalness, grounding, the 'consists in' relation, and so forth) that seem to generate the very same skeptical worries. If there were epistemic obstacles to intrinsicality qua primitive, the reductionist does not make much of an advance by replacing it with still more primitive notions that are even less familiar.

Another problem with the objection is that one can be in a position to know interesting facts regarding the essence and distribution of intrinsicality without antecedently drawing out these facts from a reductive analysis, and even if primitivism were true.

First, the truth of primitivism would itself be a highly interesting truth about the essence of intrinsicality.

Second, as we saw in sections 3 and 4.2 (and will be re-emphasized in section 5.2), there are a considerable amount of open questions about the essential nature of intrinsicality left open by the primitivist: about how many primitive notions of intrinsicality there are, which these are, and how they figure into interesting analyses of each other, or interesting analyses other notions. These would be interesting truths about intrinsicality, and appear in

23 Carroll (2009) considers, and ultimately rejects, a similar argument against those who reject that causal notions are reductively analyzable; the following discussion is inspired by (but diverges in some respects) his. 
principle resolvable (or at least, no less resolvable than similar questions for the reductionist's primitives).

Third, discussions in both philosophy and science about whether this or that notion is intrinsic often appeal only to certain diagnostics about whether a notion is intrinsic (such as whether it would either apply or fail to apply to 'carbon copies' of it, or whether it in some intuitive sense is 'independent of what is going on around it'), rather than to full-blown reductive accounts. ${ }^{24}$ There is no obvious reason why one could not be in a position to know that such diagnostics hold, even if they hold of a primitive notion.

And finally, there are a number of prominent examples of informative, explicitly primitivist theories of philosophically interesting notions, as well as a growing consensus that they are not immediately objectionable for that reason. ${ }^{25}$

\subsection{Primitivism violates plausible constraints on fundamentality}

A more promising tactic available to the reductivist is to instead argue that there are one or more antecedently motivated necessary conditions on what is fundamental-necessary conditions either on which notions are primitive, or on which facts are brute (ungrounded) facts - that a reductively unanalyzable notion of intrinsicality would fail to satisfy.

It is hard to pinpoint why so many in the literature on intrinsicality have taken primitivism to be a non-starter. But I can hazard a guess that it is rooted in the thought that if some notion of intrinsicality were primitive, then (to put it roughly at first) the fundamental level of reality would somehow fail to be minimally complete. The idea that the fundamental is constrained by minimal completeness crops up frequently in the burgeoning literature on the metaphysics of fundamentality, and is cashed out in numerous ways. Sider observes that " $[\mathrm{i}[\mathrm{t}$ is natural to assume that the fundamental must be 'complete', that the fundamental must in some sense be responsible for everything", and adds that

Completeness seems definitive of fundamentality. It would be a nonstarter to say that the fundamental consists solely of one electron: thus conceived the fundamental could not account for the vast complexity of the world we experience. (Sider 2012, p. 105)

To claim in addition that the fundamental must be minimally complete is (again roughly) to claim that the fundamental should account for the vast

24 Parsons (2001, pp. 28-29), for instance, argues that taking facts about intrinsicality as unanalyzable would not have precluded our coming to know that facts about weight are non-intrinsic.

25 See e.g. Sider (2011) on fundamentality, Williamson (2000) on knowledge, and Woodward (2002) on causation. 
complexity of the world we experience economically, and therefore without taking more notions or facts as fundamental than are necessary to account for it. Or as Schaffer puts it, "[t]he primary is (as it were) all God would need to create" in order to "generate an abundant superstructure of posterior entities" (2009, p. 351).

With this constraint on fundamentality in tow, the reductionist might argue that it would be incredible that in addition to specifying which particles exemplify which microphysical properties and relations, and decreeing which microphysical laws govern them, God would also be required to specify which microphysical properties and property exemplifications are intrinsic, and (even worse) then be required to do the same for every other subject matter that primitive notions of intrinsicality apply to. But if these facts about intrinsicality are not required to be at the fundamental level, then including them violates the constraint that the fundamental be minimally complete. So, primitivism is false.

Thus stated, the thought above is more an imprecise way of eliciting an intuition rather than a convincing argument. How might such an argument proceed? Several options present themselves. I will consider two, and argue that neither is as convincing as the intuition initially seems.

But first, some terminology is required to distinguish between two kinds of collections about which one could ask whether it is 'minimally complete' in the relevant sense. Say a collection of facts, $\Gamma$, is a complete grounding base just in case (i) every fact in $\Gamma$ is brute and (ii) if some fact is derivative, then it is ultimately grounded in a subcollection of facts in $\Gamma$. Say that $\Gamma$ is a minimally complete grounding base if and only if both (i) $\Gamma$ is a complete grounding base and (ii) no proper subcollection of facts in $\Gamma$ is a complete grounding base. ${ }^{26}$

Next, say that a collection of notions, $\Pi$, is a complete structural base just in case (i) every notion in $\Pi$ is primitive and (ii) if some notion is nonprimitive, then every fact in which it appears is ultimately grounded in some collection of facts in which only notions in $\Pi$ appear. And finally, say that $\Pi$ is a minimally complete structural base if and only if $(i) \Pi$ is a complete structural base and (ii) no proper subcollection of $\Pi$ is a complete structural base.

Notice that that the existence of a minimally complete grounding base does not logically entail the existence of a complete structural base, let alone a minimal one. (Consider a world in which some brute facts involve nonprimitive notions.) Notice also that the existence of a minimally complete structural base does not logically entail there exists a complete grounding

26 It is plausible to think that whether a fact is brute, and thus constitutes a (minimally) complete grounding base, may differ from time to time and world to world; however, for sake of readability, I will continue to suppress temporal and modal qualifications. 
base exists, let alone a minimal one. (Consider a world in which there every fact that contains only primitive notions is grounded in further facts that involve primitive notions.) Since the two notions of minimal completeness are logically non-equivalent, an argument that appeals to principles about minimal completeness must specify which notion is at issue.

Terminology in tow, I can now state and evaluate two anti-primitivist arguments. The first begins with the claim that primitive facts (i.e., facts about primitive notions) are brute facts, and then reasons to the conclusion that since there are no brute facts about intrinsicality in a minimally complete grounding base, it follows that no notion of intrinsicality is primitive. More precisely:

Argument from constraints on a minimally complete grounding base:

(1) If some notion of intrinsicality is primitive, then at least some fact about intrinsicality is brute.

(2a) If a fact is brute, it is included in a minimally complete grounding base.

(3a) A minimally complete grounding base does not include any facts about intrinsicality.

(C) No notion of intrinsicality is primitive.

The thought is this. Suppose that local intrinsicality is primitive. Then (1) states that there is some brute fact about local intrinsicality. Suppose it is the fact that the Eiffel Tower is intrinsically made of iron. So this fact is included in a minimally complete grounding base, just as (2a) states. Let $\Gamma$ be this collection of facts. But this (says the reductivist) seems absurd. Whatever facts are grounded in the fact that the Eiffel Tower is intrinsically made of iron are presumably grounded in whatever collection of facts in $\Gamma$ ground that the Eiffel Tower is made of iron. The relevant fact about intrinsicality does no interesting grounding work that facts not about intrinsicality could perform alone. Generalizing, (3a) seems true as well. Thus the anti-primitivist conclusion follows.

The second argument, like the first, begins with (1), the claim that primitive facts are brute facts. However, it then reasons to the conclusion that since at least some brute facts about intrinsicality also concern non-primitive notions, primitivism entails that notions beyond what would be included in a minimally complete structure base must also be added. More precisely:

Argument from constraints on a minimally complete structural base:

(1) If some notion of intrinsicality is primitive, then at least some fact about intrinsicality is brute. 
(2b) If some fact about intrinsicality is brute, then there are brute facts about non-primitive notions.

(3b) If there are brute facts about a notion, then the notion is included in a minimally complete structural base.

(4) No non-primitive notion is included in any minimally complete structural base.

(C) No notion of intrinsicality is primitive.

The thought is this. Suppose again that local intrinsicality is primitive, and in line with (1), suppose that the fact that the Eiffel Tower is intrinsically made of iron is a brute fact. Then in line with (2b), there is some brute fact about non-primitive notions (e.g., the property being made of iron). The motivation behind ( $3 \mathrm{~b}$ ) is the thought that one should posit a brute fact about a notion only if it is what Sider calls "pure" of non-primitive notions (2010, p. 106), and that is included in a minimally complete structural base. But as (4) states, it follows by the definition of "minimally complete structural base" that nonprimitive notions are not included in any minimally complete structural base. Thus the anti-primitivist conclusion follows.

A full evaluation of this pair of arguments would take us deep into the flourishing recent literature investigating the metaphysics of fundamentality. That said, it is worth noting that an Achilles heel to both arguments is (1), the claim the primitiveness of a notion entails that there be at least some brute facts about it.

There are at least three observations the primitivist might make. First, the primitivist may note that no immediate logical or conceptual incoherence to results from maintaining that a primitive notion appear in no brute facts. It is widely recognized that a fact's being grounded does not entail that notions involved in are reductively analyzable. ${ }^{27}$ But if the grounding of a fact does not entail there be a reductive analysis of the notions that fact is about, it is unclear at best why the lack of a reductive analysis of the notions involved in some fact would entail that this fact lacks a ground.

Second, arguably there are counterexamples to (1)'s truth which concern notions other than notions of intrinsicality. Indeed, several philosophers have argued that the notion of grounding - the very same one that appears in many recent attempts at reductive analysis - is precisely such a case. ${ }^{28}$

27 Thus Louis deRosset observes that "the layered structure given by grounding explanations, unlike the layered structure given by reductions, is consistent with the phenomenon known as multiple realizability. A fact $P$ can be explained by a fact $Q$, even if it is possible that something other than $Q$ explain $P$, and $Q$ not obtain at all" (2013, p. 5). Indeed, a fact's being grounded at one time and world seems compatible with it obtaining at some other time and world at which that fact is not grounded at all. There is no obvious incoherence in taking facts about conscious phenomena to be grounded in physical properties in the actual world yet brute in other, 'more distant' counterfactual possibilities, for instance.

28 See Bennett (2011) and deRosset (2013) for arguments to this effect.

24 ALEXANDER SKILES 
Third nothing appears to prevent the primitivist from supplying a general principle regarding how facts about the primitive notions of intrinsicality get systematically grounded in facts that involve no notions of intrinsicality at all. One might, for instance, take the fact that a thing, $x$, has a property $F$ to be grounded in facts about the essential nature of $F$, or about the essential nature of $[x$ is $F]$. Or the primitivist might simply co-opt the collections of facts that attempted reductive analyses have offered as sufficient conditions for intrinsicality. She might claim that although these accounts collectively state sufficient grounds for $x$ to be $F$ intrinsically, depending on the kind of thing or property at issue, nonetheless they do not constitute any necessary conditions for something to have properties intrinsically, holding across all possible circumstances.

Arguments that appeal to constraints on fundamentality appear to be the most promising for supporting reductionism. However, much more needs to be said in their defense.

\section{Conclusion}

This chapter was intended to be exploratory rather than definitive; limited rather than exhaustive. I made no attempt to develop a complete and detailed primitivist theory; rather, I have sketched the major options that confront the primitivist, and presented some open questions that must be settled in order to determine whether the view is correct. Nor do I claim to have surveyed all the arguments either for or against primitivism about intrinsicality, nor have I determined which of these are sound.

Although my aims have been modest, nonetheless I hope to have shown at least the following. There is a rich terrain of primitivist theories to map out. The issues that they border on are among the deepest and most important in contemporary metaphysics. Those attracted to primitivism have quite a bit of territory to explore. And those who still hold hope in reductive approaches to intrinsicality have much more to say about why we should continue to stick to the faith. ${ }^{29}$

29 Many thanks to Mark Barber, Philipp Blum (née Keller), Pablo Carnino, Maya Eddon, Robert Francescotti, Akiko Frischhut, Dan Marshall, Michaela McSweeney, Josh Parsons, Graham Peebles, Aaron Segal, Jennifer Wang, and Tobias Wilsch for helpful conversations covering the topics of this paper. This article was written while funded by the Swiss National Science Foundation as a member of the Sinergia research project 'Intentionality as the Mark of the Mental' (project \#: CRSI11-127488), and I am grateful for its generous support. 


\section{References}

Bader, Ralf (forthcoming). "Towards a Hyperintensional Theory of Intrinsicality", The Journal of Philosophy.

Bennett, Karen (2011). "By Our Bootstraps", Philosophical Perspectives 25: 27-41.

Carroll, John (2009). "Anti-Reductionism”, in H. Beebee, C. Hitchcock, P. Menzies (eds.), The Oxford Handbook of Causation (pp. 279-298). Oxford: Oxford University Press.

Chisholm, R. (1976). Person and Object. LaSalle, IL: Open Court Press.

Correia, Fabrice (2006). "Generic Essence, Objectual Essence, and Modality”, Noûs 40: 753-767.

Daly, Chris (2012). "Scepticism about Grounding", in F. Correia and B. Schnieder (eds.) (2012), Metaphysical Grounding: Understanding the Structure of Reality (pp. 81-100). Cambridge: Cambridge University Press.

Denby, A. David (2006). "The Distinction between Intrinsic and Extrinsic Properties", Mind 115: 1-17.

(2010). "Intrinsic and Extrinsic Properties: A reply to HoffmannKolss", Mind, 119: 773-782.

Dennett, D. (1988). "Quining Qualia”, in A. J. Marcel and E. Bisiach (eds.), Consciousness in Contemporary Science (pp.42-77). New York: Oxford University Press.

DePaul, Michael and William Ramsey (eds.) (1998). Rethinking Intuition: The Psychology of Intuition and Its Role in Philosophical Inquiry. Lanham, Maryland: Rowman \& Littlefield.

deRosset, Louis (2013). "Grounding Explanations", Philosophers' Imprint 13: 1-26.

Dorr, Cian (2004), "Non-Symmetric Relations", in D. Zimmerman (ed.), Oxford Studies in Metaphysics, vol. 1 (pp. 155-192). Oxford: Clarendon Press.

(2005). "What We Disagree About When We Disagree About Ontology”, in. Kalderon (ed.), Fictionalism in Metaphysics (pp. 234-286). Oxford: Oxford University Press.

(2008), "There Are No Abstract Objects", in T. Sider, J. Hawthorne and D. Zimmerman (eds.), Contemporary Debates in Metaphysics (pp. 32-64). Malden, MA: Blackwell. (manuscript), "To Be an F is To Be a G".

Eddon, Maya (2010). Three Arguments From Temporary Intrinsics", Philosophy and Phenomenological Research 81: 605-619.

(2011). "Intrinsicality and Hyperintensionality", Philosophy and Phenomenological Research 82: 314-336.

Ellis, Brian (1991). "Scientific Essentialism". Paper presented to the 1991 conference of the Australasian Association for the History and Philosophy of Science.

Figdor, Carrie (2008). "Intrinsically/Extrinsically", The Journal of Philosophy 105: 691-718.

Fine, Kit (1994). "Essence and Modality", Philosophical Perspectives 8: 1- 
16.

(1995). "The Logic of Essence", Journal of Philosophical Logic 24: $241-273$.

(2010). "Towards a Theory of Part", The Journal of Philosophy 107: 559-589.

Francescotti, Robert (1999). "How to Define Intrinsic Properties", Noûs 33: 590-609.

Hawthorne, John (2009). "Superficialism in Ontology", in D. Chalmers, D. Manley, and R. Wasserman (eds.), Metametaphysics: New Essays on the Foundations of Ontology (pp. 213-230). Oxford: Oxford University Press. Hofweber, Thomas (2009). "Ambitious, Yet Modest, Metaphysics", in D. Chalmers, D. Manley, and R. Wasserman (eds.), Metametaphysics: New Essays on the Foundations of Ontology (pp. 260-289). Oxford: Oxford University Press.

Hoffmann-Kolss, Vera (2010a). The Metaphysics of Extrinsic Properties. Frankfurt: Ontos-Verlag.

(2010b). "Denby on the Distinction Between Intrinsic and Extrinsic Properties", Mind 119: 763-772.

Hudson, H. (2001). A Materialist Metaphysics of the Human Person. Ithaca: Cornell University Press.

Humberstone, I. L. (1996). "Intrinsic/Extrinsic", Synthese 108: 205-267.

Jacobs, Jonathan D. (2010). "A Powers Theory of Modality-or, How I Learned to Stop Worrying and Reject Possible Worlds", Philosophical Studies 151: 227-248.

Kim, J. (1982). "Psychophysical Supervenience", Philosophical Studies 41: 51-70.

King, Jeffrey (1998). "What is a Philosophical Analysis?", Philosophical Studies 90: 155-179.

Ladyman, James and Don Ross (2007). "Ontic Structural Realism and the Philosophy of Physics", in J. Ladyman and D. Ross (eds.), Every Thing Must Go: Metaphysics Naturalized (pp. 130-189). Oxford: Oxford University Press.

Langton, Rae and David Lewis (1998). "Defining 'Intrinsic'”, Philosophy and Phenomenological Research 58: 333-45.

Lewis, David (1983a). “Extrinsic Properties”, Philosophical Studies 44: 197200.

(1983b). "New Work for a Theory of Universals", Australasian Journal of Philosophy 61: 343-77.

(1986a). On the Plurality of Worlds. Oxford: Blackwell.

Lowe, E. J. (1998). The Possibility of Metaphysics: Substance, Identity and Time. Oxford: Oxford University Press.

(2012). "Asymmetrical Dependence in Individuation”, F. Correia and B. Schnieder (eds.), Metaphysical Grounding: Understanding the Structure of Reality (pp. 214-233). Cambridge: Cambridge University Press.

Marshall, Dan (2009). "Can 'Intrinsic' Be Defined Using Only Broadly Logical Notions?", Philosophy and Phenomenological Research 78: 646-672. (2012). "Analyses of Intrinsicality in Terms of Naturalness", Phil- 
osophy Compass 7: 531-542.

(forthcoming-a). "Analyses of Intrinsicality Without Naturalness",

Philosophy Compass.

(forthcoming-b). "Intrinsicality and Grounding", Philosophy and Phenomenological Research.

Melia, Joseph (2005). "Truthmaking Without Truthmakers", in H. Beebee and J. Dodd (eds.), Truthmakers: The Contemporary Debate (pp. 67-84). Oxford: Oxford University Press.

McDaniel, Kris (2004). "Modal Realism with Overlap", Australasian Journal of Philosophy 82: 137-152.

Parsons, Josh (2000), "Must a Four-Dimensionalist Believe in Temporal Parts?", The Monist 83: 399-418. (2001), Theories of Persistence. Ph. D. thesis, Canberra: Australian National University. (2004). "Distributional Properties", in F. Jackson and G. Priest (eds.), Lewisian Themes: The Philosophy of David K. Lewis (pp. 173180). Oxford: Oxford University Press.

Rayo, Agustín (2013). The Construction of Logical Space. Oxford: Oxford University Press.

Rosen, Gideon (2010). "Metaphysical Dependence: Grounding and Reduction", in B. Hale and A. Hoffmann (eds.), Modality: Metaphysics, Logic, and Epistemology (pp. 109-136). Oxford: Oxford University Press.

Schaffer, Jonathan (2009). "On What Grounds What", in D. Chalmers, D. Manley, and R. Wasserman (eds.), Metametaphysics: New Essays on the Foundations of Ontology (pp. 347-383). Oxford: Oxford University Press.

Schroeder, Mark (2005). "Realism and Reduction: The Quest for Robustness", Philosophers' Imprint 5: 1-18.

Sider, Theodore (1993). Naturalness, Intrinsicality, and Duplication. Ph.D thesis, Amherst: the University of Massachussetts at Amherst.

__ (1996). "Intrinsic Properties", Philosophical Studies 83: 1-27.

- (2001). Four-Dimensionalism: An Ontology of Persistence and Time. Oxford: Oxford University Press.

__ (2007). "Parthood", Philosophical Review 116: 51-91.

- (2011). Writing the Book of the World. Oxford University Press.

Skow, Bradford (2007). “Are Shapes Intrinsic?”, Philosophical Studies 133: 111-130.

Taylor, B. (1993). "On Natural Properties in Metaphysics", Mind 102, pp. 81-100.

Trogdon, Kelly (2009). "Monism and Intrinsicality", Australian Journal of Philosophy 87: 127-148.

Vallentyne, Peter (1997). "Intrinsic Properties Defined", Philosophical Studies 88: 209-219.

Wasserman, Ryan (2005). "Humean Supervenience and Personal Identity", The Philosophical Quarterly 55: 582- 593.

Weatherson, Brian (2001). "Intrinsic Properties and Combinatorial Principles", Philosophy and Phenomenological Research 63: 365-80.

Weatherson, Brian and Dan Marshall (2012). "Intrinsic vs. Extrinsic Properties", in E. Zalta (ed.), The Stanford Encyclopedia of Philosophy, 
<http://plato.stanford.edu/archives/spr2013/entries/intrinsic-extrinsic/> Wedgwood, Ralph (2007). The Nature of Normativity. Oxford: Oxford University Press.

Williams, J. R. G. (2013). "Part-Intrinsicality", Noûs 47: 413-452.

Williamson, Timothy (2000). Knowledge and its Limits. Oxford: Oxford University Press.

Witmer, Gene, William Butchard and Kelly Trogdon (2005). "Intrinsicality without Naturalness", Philosophy and Phenomenological Research 70: 326-50.

Woodward, James (2003). Making Things Happen: A Theory of Causal Explanation. Oxford: Oxford University Press.

Yablo, Stephen (1999). "Intrinsicness", Philosophical Topics 26: 479-505. 